\title{
A COMPETENCY EVALUATION METHOD OF HUMAN RESOURCES MANAGERS BASED ON MULTI-GRANULARITY LINGUISTIC VARIABLES AND VIKOR METHOD
}

\author{
Peide Liu ${ }^{1}$, Xingying $\mathrm{Wu}^{2}$ \\ School of Management Science and Engineering, Shandong University of Finance and Economics, \\ Jinan 250014, China \\ E-mails: ${ }^{1}$ peide.liu@gmail.com (corresponding author); ${ }^{2}$ wuxingying_4499@126.com
}

Received 10 December 2010; accepted 24 October 2011

\begin{abstract}
According to the characteristics of human resources managers' competency and the research of relevant literatures at home and abroad, a competency evaluation method of human resources managers based on multi-granularity linguistic variables and VIKOR method is proposed. Firstly, the evaluation indicator system based on the competency of human resources managers is constructed. Then, by converting the evaluation information of different experts in different granularity to the same granularity by two-semantics, the comprehensive evaluation values can be obtained by integrating information of different experts. Further, the objective weights of the evaluation indexes were determined by the entropy method and an evaluation method were proposed based on the VIKOR method. Finally, an application example is given to illustrate the evaluation procedures of the developed approach and to demonstrate their practicality and effectiveness.
\end{abstract}

Keywords: human resources managers, entropy, VIKOR, multi-granularity, two-semantics.

Reference to this paper should be made as follows: Liu, P.; Wu, X. 2012. A competency evaluation method of human resources managers based on multi-granularity linguistic variables and VIKOR method, Technological and Economic Development of Economy 18(4): 696-710.

JEL Classifcation: D81, N55, C44, O13.

\section{Introduction}

With the arrival of the global economic integration age, the scope of the enterprise competition expanded rapidly and the degree of competition increased unprecedentedly. Ultimately the competition of enterprise is the competition of talent. Modern human resources managers thought that human resources managers were the core of all the management 
jobs. It emphasized on employees as potential resources and emphasized on the motivation and development of staff (Wang 2007). And human resources managers of enterprises were the organizers and actors in human resources management. They played an important role in human resources management. The competence level of human resources managers was decisive on whether human resources management of company was effective (Castrogiovanni, Kidwell 2010; Davidson, Wang 2011; Hashim 2010; Sharabi 2010). The evaluation results of human resources managers' competency could be used as (Zhang et al. 2005): (1) the basis for selecting and training human resources managers; (2) the basis of making the training policies and measures (3) providing a direction for the self-development of human resources manager.

The evaluation of human resources managers' competency is not only the academic focus, but also an important problem which needed to be solved by many enterprises. Currently, the evaluation of human resources managers' competency had aroused widely concern between academia and industrial circles and gotten many research results. Wang and Hwang (2011) used the analytical hierarchy process (AHP) to analyze the key factors in evaluating and screening managers in Taiwan. Zhang et al. (2005) proposed a subjective and objective evaluation method of human resources managers' competency according to the features and job description of human resources managers, built subjective indicators, including educational background and intellectual structure, personal integrity, ability to manage enterprise culture, ability to manage change, ability to manage knowledge, ability to transmit human resource management practices and objective indicators of multi-knowledge test, including strategic management, organization behaviorist and human resources management. Zhou and Zhang (2009) brought forward competency testing indicator system of enterprise's human resources managers according to the competency testing indicator system raised by American psychologist Dr McClelland, built a fuzzy comprehensive evaluation model of enterprise's human resources management based on fuzzy math and AHP and executed empirical studies; Liu et al. (2009) used the interview method and questionnaire survey to gather data, dealt with data by Exploratory Factor Analysis and Analysis of Variance, and finally worked out a competency model of Chinese enterprises' human resources managers, which made up of three-factors and 15 items; Zhao (2008) firstly analyzed fuzzy factor of competency-based talent selection process and built multi-criteria fuzzy decision-making math model; Chen (2006) built Chinese enterprises' competency model of human resources managers including competency of functional management, competency of management of change, competency of staff management and competency of strategic management by questionnaire survey method; Spencer et al. (1994) believed that human resources managers needed flexible competency, business innovation, interpersonal understanding, empowerment and team growth; Research project of Ulrich et al.(1995) stated clearly, participants felt that it proved competency of human resources managers more efficiently in term of business knowledge, human resources implement and management of change. Questionnaire survey results by $\mathrm{Gu}$ and $\mathrm{Zhu}$ (2001) showed that, human resources managers of Shanghai enterprises believed that the most important eight-point competencies in turn were worthy of trust, problem-solving, ability to identify people, communicative competency, human resources expertise, learning ability, service awareness and analysis ability. Ma and Cai (2007) arose 
ten-point competencies of human resources managers, including learning ability, systems thinking ability, service ability, ability to integrate human resources, strategic performance management, human resources crisis management capacity, the operational capabilities of information technology, global capability, resilience and executive ability. Mussari and Ruggiero (2010) thought that personnel management has been one of the areas of greatest innovation within the management reform process in western countries over the last two decades, and analyzed the public managers' performance evaluation systems and public value creation from behavioral and economic aspects.

From the past few years, Decision theory and methods based on fuzzy information have been rapid development (Zavadskas, Turskis 2011; Han, Liu 2011; Liu 2009; Liu, Zhang 2011), especially the research based on evaluation information of linguistic form had been the concern of many scholars. In order to facilitate the experts more accurately to express their subjective judgments and further improve the efficiency and quality of group decision making and the availability and flexibility of network environment group decision support system, the group decision-making research considered different granularity of linguistic information given by the experts attracted the attention of scholars. The so-called multi-granularity linguistic assessment information referred that different experts used different linguistic evaluation set for the same decision making problem to give their own set of linguistic forms of assessment information in the group decision-making, and the selected set of linguistic evaluation contained many differences on the number of linguistic phrases and corresponding phrase semantic of membership functions. Group decision-making process based on different granularity linguistic evaluation information mainly included group preference information gathered and group consistency analysis. Nowadays, research and exploration of group decision-making method based on different granularity linguistic assessment information had caused some scholars' interest and concerns of the United Kingdom, Spain and other countries. But the results were rare. As noted, most of the existing research results involved gathering group decision information and converting the different granularity linguistic evaluation information. About group consistency analysis, Herrera et al. (2005) studied the group consistency analysis method of the different granularity linguistic judgment matrix. This method firstly converted different granularity linguistic assessment information offered by decision-makers into fuzzy information evaluation set expressed by two- semantics based on two- semantics linguistic method, then made the group consistency analysis.

From the above, the current evaluation of human resources managers' competency was mainly qualitative evaluation and lacks of quantitative evaluation method. As the evaluation of human resources managers' competency was mostly qualitative indicators which generally used the "excellent", "good", "general" and "poor", and other linguistic variables. At the same time, because different experts may adopt different linguistic evaluation sets, therefore assessment information also had multi-granularity properties. Based on multi-granularity linguistic assessment information, the paper launched a research for evaluation of human resource managers' competency, and proposed an evaluation model of human resources managers' competency based on multi-granularity linguistic variables and VIKOR method. 


\section{The evaluation index of human resource managers' competency}

The evaluation index of human resources managers' competency is the basis of human resources managers' competency evaluation. We built a preliminary evaluation indicator system and considered the relevant factors by documentary research method, in complying with the principle of human resources managers' competency evaluation, such as comparability, objectivity, comprehensiveness, reliability, and flexibility. Then the human resources managers' evaluation index systems are constructed based on the opinions of experts, human resources managers and human resources assess company (shown in Table 1).

Table 1. Human resources manager competency evaluation system

\begin{tabular}{ll}
\hline \multicolumn{1}{c}{ Contents } & \multicolumn{1}{c}{ Indicators } \\
\hline A1 Moral level & B1 Honesty Degree \\
& B2 Sense of responsibility \\
& B3 Confidentiality \\
\hline A2 Capability level & B4 Learning ability \\
& B5 Ability to manage change \\
& B6 Service capacity \\
& B7 Communication skills \\
& B8 The operational capabilities of information technology \\
& B9 Implementing competence \\
\hline A3 Knowledge level & B10 Business knowledge \\
& B11 Knowledge of laws and regulations \\
\hline A4 Professional quality Level & B12 Recruitment Management \\
& B13 Training Management \\
& B14 Performance Management \\
& B15 Compensation Management \\
\hline A5 Health level & B16 Mental states \\
& B17 Mass Psychology \\
\hline
\end{tabular}

\section{The VIKOR evaluation model based on the linguistic variables of the different granularity}

\subsection{Describing problem of human resources managers' competency evaluation}

Suppose that there are $m$ human resources managers (evaluation objects) $A=\left(A_{1}, A_{2}, \cdots, A_{m}\right), n$ evaluation indicators $B=\left(B_{1}, B_{2}, \cdots, B_{n}\right)$, p evaluation experts $E=\left(e_{1}, e_{2}, \cdots, e_{p}\right)$, experts weight is $\omega=\left(\omega_{1}, \omega_{2}, \cdots, w_{p}\right)$, and $\sum_{k=1}^{p} \omega_{k}=1$.The evaluation indicator weight is $W=\left(w_{1}, w_{2}, \cdots, w_{n}\right)$, 
$\sum_{j=1}^{n} w_{j}=1$ and $w_{j}$ is unknown. Suppose that the evaluation index values given by the $k$ th evaluation expert compose a matrix of $R^{k}=\left[r_{i j}^{k}\right]_{m \times n}, r_{i j}^{k}$ is the $j$ th index evaluation value of the $i$ th evaluation object by the $k$ th evaluation expert. $S_{i}^{k}=\left(s_{0}^{k}, s_{1}^{k}, s_{2}^{k}, \ldots, s_{q_{k}-1}^{k}\right)$ is the linguistic evaluation set which granularity is $q_{k}$ adopted by the $i$ th expert. This evaluation question will rank the $m$ human resources managers.

\subsection{Normalize evaluation sets of different granularity by two-semantics}

Two-semantics is a concept based the symbol translation and method using a two-semantics $(s, \alpha)$ to represent linguistic assessment information. Among them, $s$ is a linguistic phrase of predetermined linguistic set, $A_{i}$ is the difference between linguistic information calculated and the closest linguistic phrase of initial linguistic set, which is a value in interval $[-0.5,0.5]$. The following are the related definitions of two-semantics (Zhou, Zhang 2009; Liu et al. 2009);

Definition 1. Suppose that $s_{i} \in S$ is a linguistic phrase. Then we can get the corresponding two-semantics by the following translation function $\theta$ :

$$
\begin{gathered}
\theta: S \rightarrow S \times[-0.5,0.5), \\
\theta\left(s_{i}\right)=\left(s_{i}, 0\right), s_{i} \in S .
\end{gathered}
$$

Definition 2. (Herrera, Martinez 2000, 2001; Herrera et al. 2005): Let $S=\left(s_{0}, s_{1}, \cdots, s_{g}\right)$ be a linguistic term set, $\beta$ is a real number in $[0, g]$, and it represents the calculating result of aggregation for the elements in $S$, then two-semantics corresponding to the elements in $S$ can be gotten from the following function:

$$
\begin{gathered}
\Delta:[0, g] \rightarrow S \times[-0.5,0.5), \\
\Delta(\beta)=\left(s_{i}, \alpha\right), \\
\text { here, } i=\operatorname{round}(\beta), \alpha=\beta-i, \alpha \in[-0.5,0.5),
\end{gathered}
$$

where, round(.) is an integer operator of rounding.

Definition 3. (Herrera, Martinez 2000, 2001; Herrera et al. 2005): Let $S=\left(s_{0}, s_{1}, \cdots, s_{g}\right)$ be a linguistic set, $\left(s_{i}, \alpha\right)$ be a two-semantics and then there is an inverse function $\Delta^{-1}$ which can convert the two-semantics into corresponding real number $\beta \in[0, g]$, that is:

$$
\begin{gathered}
\Delta^{-1}: S \times[-0.5,0.5) \rightarrow[0, g-1], \\
\Delta^{-1}\left(s_{i}, \alpha\right)=i+\alpha=\beta .
\end{gathered}
$$

In order to eliminate the influence of different granularity, the different granularity linguistic matrix $R=\left(r_{i j}^{k}\right)_{m \times n}$, should be converted to the same granularity. Suppose that $T$ is the granularity after converted. The conversion method is shown as follows.

$$
r_{i j}^{k}=\frac{r_{i j}^{k}}{q_{k}} T \forall i \in m ; \forall j \in n ; \forall k \in p,
$$

where $q_{k}$ is the granularity adopted by expert $k$. 
Based on above definitions, it is easy to give the related calculating model of two-semantics, which including: comparing of the two-semantics, inverse operator and aggregation operator.

1. The comparing of the two-semantics. Let $\left(s_{i}, \alpha_{1}\right)$ and $\left(s_{j}, \alpha_{2}\right)$ be any two two-semantics, and there are such rules:

If $i>j$, then $\left(s_{i}, \alpha_{1}\right)>\left(s_{j}, \alpha_{2}\right)$, which means $\left(s_{i}, \alpha_{1}\right)$ is superior to $\left(s_{j}, \alpha_{2}\right)$;

If $i=j$ and $\alpha_{1}=\alpha_{2}$, then $\left(s_{i}, \alpha_{1}\right)=\left(s_{j}, \alpha_{2}\right)$, which means $\left(s_{i}, \alpha_{1}\right)$ is the same as $\left(s_{j}, \alpha_{2}\right)$;

If $i=j$ and $\alpha_{1}>\alpha_{2}$, then $\left(s_{i}, \alpha_{1}\right)>\left(s_{j}, \alpha_{2}\right)$, which means $\left(s_{i}, \alpha_{1}\right)$ is superior to $\left(s_{j}, \alpha_{2}\right)$;

If $i=j$ and $\alpha_{1}<\alpha_{2}$, then $\left(s_{i}, \alpha_{1}\right)<\left(s_{j}, \alpha_{2}\right)$, which means $\left(s_{i}, \alpha_{1}\right)$ is inferior to $\left(s_{j}, \alpha_{2}\right)$.

2. There is an inverse operator Neg:

$\operatorname{Neg}\left(s_{i}, \alpha\right)=\Delta\left(g-\left(\Delta^{-1}\left(s_{i}, \alpha\right)\right)\right)$.

3. If $\left(s_{i}, \alpha_{1}\right) \geq\left(s_{j}, \alpha_{2}\right)$, then $\max \left\{\left(s_{i}, \alpha_{1}\right),\left(s_{j}, \alpha_{2}\right)\right\}=\left(s_{i}, \alpha_{1}\right)$;

and if $\left(s_{i}, \alpha_{1}\right) \leq\left(s_{j}, \alpha_{2}\right)$, then $\min \left\{\left(s_{i}, \alpha_{1}\right),\left(s_{j}, \alpha_{2}\right)\right\}=\left(s_{i}, \alpha_{1}\right)$.

4. The distance between two two-semantics:

The distance between two two-semantics $A:\left(s_{i}, \alpha_{1}\right)$ and $B:\left(s_{j}, \alpha_{2}\right)$ is:

$$
d(A, B)=\left|\Delta^{-1}\left(s_{i}, \alpha_{1}\right)\right|-\left|\Delta^{-1}\left(s_{j}, \alpha_{2}\right)\right| .
$$

Theorem 1: For any three-semantics $A:\left(s_{i}, \alpha_{1}\right), B:\left(s_{j}, \alpha_{2}\right)$ and $C:\left(s_{k}, \alpha_{3}\right)$, according to formula (5), the distance $d(A, B)$ between $A$ and $B$ satisfies the following conditions:

(1) $A=B \Leftrightarrow d(A, B)=0$,

(2) $d(A, B)=d(B, A)$,

(3) $d(A, B)+d(B, C) \geq d(A, C)$.

\subsection{Integrating evaluation information of each expert}

According to the different evaluation index values which were given by different experts under different attribute, we can get the collective attribute values.

The combining steps are shown as follows:

$$
r_{i j}=\sum_{k=1}^{p} \omega_{k} r_{i j}^{k} .
$$

\subsection{Using the entropy weight method to calculate attribute weights (Zhao 2008; Chen 2006)}

(1) Calculate $u_{i j}$ (the proportion of the $i$ th object value under the $j$ th index):

$$
u_{i j}=\frac{r_{i j}}{\sum_{i=1}^{m} r_{i j}} .
$$

(2) Calculate entropy $e_{j}$ of the $j$ th index:

$$
e_{j}=-k \sum_{i=1}^{m} u_{i j} \ln u_{i j},
$$

where $k=\frac{1}{\ln m}, m$ is the number of human resources managers 
(3) Calculate the weight $w_{j}$ :

$$
w_{j}=\frac{1-e_{j}}{\sum_{j=1}^{n}\left(1-e_{j}\right)}
$$

\subsection{Select the best candidate using VIKOR method}

VIKOR, which Serbian name was "VlseKriterijumska Optimizacija I Kompromisno Resenje, means multi-criteria optimization and compromise solution" (Chu et al. 2007), was developed by Opricovic (1998), Opricovic and Tzeng (2002). The VIKOR method was developed for multi-criteria optimization of complex systems (Opricovic, Tzeng 2004). This method focused on ranking and selecting from a set of alternatives, and determined compromise solutions for a problem with conflicting criteria, which can help the decision makers to reach a final decision. Here, the compromise solution is a feasible solution which is closest to the ideal and a compromise mean established by mutual concessions (Opricovic, Tzeng 2007).

Assuming that each alternative is evaluated according to each attribute function, the compromise ranking could be performed by comparing the measure of closeness to the ideal alternative. The multi-attribute measure for compromise ranking is developed from the $L p-$ metric used as an aggregating function in a compromise programming method (Yu 1973; Zeleny 1982). The various $m$ alternatives are denoted as $A_{1}, A_{2}, \cdots, A_{m}$. For alternative $A_{i}$, the rating of the $j$ th aspect is denoted by $f_{i j}$, i.e. $f_{i j}$ is the value of $j$ th attribute function for the alternative $A_{i} ; n$ is the number of attribute.

Development of the VIKOR method is started with the following form of $L p-$ metric :

$$
L_{p, i}=\left\{\sum_{j=1}^{n}\left[w_{j}\left(f_{j}^{*}-f_{i j}\right) /\left(f_{j}^{*}-f_{j}^{-}\right)\right]^{p}\right\}^{1 / p} \quad 1 \leq p \leq \infty ; i=1,2, \cdots m .
$$

In the VIKOR method, $L_{1, i}$ (as $S_{i}$ ) and $L_{\infty, i}$ (as $R_{i}$ ) are used to formulate ranking measure. The solution obtained by $\min S_{i}$ is with a maximum group utility ("majority" rule), and the solution obtained by $\min R_{i}^{i}$ is with a minimum individual regret of the "opponent".

The compromise ranking algorithm of the VIKOR method has the following steps:

(1) Determine the best $f_{j}^{*}$ and the worst $f_{j}^{-}$values of all attribute functions, $j=1,2, \cdots, n$ If the $j$ th function represents a benefit (if it is cost attribute, it can be converted into benefit by standardizing), then:

$$
f_{j}^{*}=\max _{i}\left(f_{i j}\right), f_{j}^{-}=\min _{i}\left(f_{i j}\right) .
$$

(2) Compute the values $S_{i}$ and $R_{i}, i=1,2, \cdots, m$, by the relations:

$$
\begin{aligned}
& S_{i}=\sum_{j=1}^{n}\left[w_{j}\left(f_{j}^{*}-f_{i j}\right) /\left(f_{j}^{*}-f_{j}^{-}\right)\right], \\
& R_{i}=\max _{j}\left[w_{j}\left(f_{j}^{*}-f_{i j}\right) /\left(f_{j}^{*}-f_{j}^{-}\right)\right],
\end{aligned}
$$

where $w_{j}$ are the weights of criteria, expressing their relative importance. 
(3) Compute the values $Q_{i}, i=1,2, \cdots, m$, by the following relation:

$$
Q_{i}=v \frac{\left(S_{i}-S^{*}\right)}{\left(S^{-}-S^{*}\right)}+(1-v) \frac{\left(R_{i}-R^{*}\right)}{\left(R^{-}-R^{*}\right)},
$$

where, $S^{*}=\min _{i} S_{i}, S^{-}=\max _{i} S_{i}, R^{*}=\min _{i} R_{i}, R^{-}=\max _{i} R_{i}$,

$v$ is introduced as weight of the strategy of "the majority of criteria" (or "the maximum group utility"), here suppose that $v=0.5$.

(4) Rank the alternatives. Sort the value $Q$ in decreased order. The position in the front is better than in the behind.

\section{Application research}

A company planned to select one person to be human resource manager from four candidates $\left(a_{1}, a_{2}, a_{3}, a_{4}\right)$. In order to improve the accuracy of the evaluation, three experts were invited to evaluate these candidates, they were the vice president in charge of the company human resources, a university professor and an expert of human resource assessment company. Suppose that the 3 person scored independent and they had the same evaluation weight. The index evaluation values given by three experts were shown in table 2, table 3 and table 4 respectively. Expert 1 used 5 level linguistic variables $=\{$ very poor, poor, medium, good, very good $\}$, expert 2 used 7 level linguistic variables $=\{$ very bad, very poor, poor, medium, good, quite good, very good $\}$, Experts 3 used 9 level linguistic variables $=\{$ very bad, very poor, less poor, poor, medium, good, better, very good, best\}. Our goal is to select a human resource manager based on these evaluation values. (For convenient representation, we only use the subscript of linguistic variables, for example, $s_{4}$ was expressed by 4 ).

Table 2. The index evaluation value by expert 1 ( 5 level linguistic variables)

\begin{tabular}{cccccccccccccccccc}
\hline & B1 & B2 & B3 & B4 & B5 & B6 & B7 & B8 & B9 & B10 & B11 & B12 & B13 & B14 & B15 & B16 & B17 \\
\hline$a 1$ & 2 & 3 & 4 & 1 & 2 & 3 & 4 & 2 & 4 & 3 & 2 & 4 & 2 & 2 & 2 & 4 & 2 \\
\hline$a 2$ & 1 & 2 & 4 & 2 & 3 & 4 & 2 & 1 & 4 & 2 & 3 & 2 & 4 & 4 & 2 & 4 & 1 \\
\hline$a 3$ & 1 & 3 & 4 & 2 & 1 & 4 & 1 & 3 & 2 & 4 & 2 & 1 & 4 & 2 & 1 & 3 & 4 \\
\hline$a 4$ & 1 & 3 & 4 & 2 & 4 & 2 & 4 & 3 & 2 & 1 & 2 & 3 & 2 & 4 & 2 & 3 & 4 \\
\hline
\end{tabular}

Table 3. The index evaluation value by expert 2 (7 level linguistic variables)

\begin{tabular}{cccccccccccccccccc}
\hline & B1 & B2 & B3 & B4 & B5 & B6 & B7 & B8 & B9 & B10 & B11 & B12 & B13 & B14 & B15 & B16 & B17 \\
\hline$a 1$ & 2 & 4 & 6 & 3 & 4 & 3 & 2 & 4 & 5 & 6 & 1 & 3 & 4 & 6 & 3 & 2 & 4 \\
\hline$a 2$ & 2 & 4 & 2 & 5 & 6 & 3 & 3 & 1 & 4 & 5 & 3 & 5 & 6 & 5 & 2 & 1 & 3 \\
\hline$a 3$ & 1 & 3 & 3 & 2 & 1 & 4 & 5 & 3 & 2 & 6 & 5 & 4 & 3 & 2 & 5 & 4 & 5 \\
\hline$a 4$ & 4 & 3 & 4 & 5 & 1 & 2 & 3 & 5 & 4 & 3 & 2 & 6 & 6 & 5 & 5 & 2 & 5 \\
\hline
\end{tabular}


Table 4. The index evaluation value by expert 3 (9 level linguistic variables)

\begin{tabular}{cccccccccccccccccc}
\hline & B1 & B2 & B3 & B4 & B5 & B6 & B7 & B8 & B9 & B10 & B11 & B12 & B13 & B14 & B15 & B16 & B17 \\
\hline$a 1$ & 6 & 7 & 4 & 8 & 2 & 4 & 2 & 8 & 6 & 2 & 1 & 5 & 6 & 7 & 2 & 5 & 2 \\
\hline$a 2$ & 1 & 2 & 4 & 6 & 7 & 8 & 4 & 6 & 3 & 5 & 5 & 6 & 7 & 3 & 1 & 5 & 7 \\
\hline$a 3$ & 1 & 3 & 5 & 8 & 7 & 5 & 4 & 3 & 2 & 6 & 7 & 8 & 3 & 7 & 1 & 5 & 8 \\
\hline$a 4$ & 5 & 4 & 6 & 3 & 1 & 6 & 7 & 8 & 9 & 4 & 3 & 2 & 5 & 6 & 7 & 8 & 5 \\
\hline
\end{tabular}

Decision steps are shown as follows:

(1) Converting evaluation information given by three experts into evaluation information expressed by 9 level linguistic set (shown as Table 5, Table 6, Table 7 respectively).

Table 5. The index evaluation value by expert 1

\begin{tabular}{|c|c|c|c|c|c|c|c|c|c|c|c|c|c|c|c|c|c|}
\hline & B1 & B2 & B3 & B4 & B5 & B6 & B7 & B8 & B9 & B10 & B11 & B12 & B13 & B14 & B15 & B16 & B17 \\
\hline ह & \begin{tabular}{l} 
f! \\
$\stackrel{1}{1}$ \\
\multirow{j}{*}{}
\end{tabular} & \begin{tabular}{l}
\multirow{+}{0}{} \\
0 \\
0
\end{tabular} & 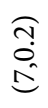 & $\begin{array}{l}\text { กุ. } \\
\hat{1} \\
\hat{d}\end{array}$ & $\begin{array}{l}\text { f! } \\
\stackrel{1}{1} \\
\dot{J}\end{array}$ & $\begin{array}{l}\text { ft } \\
0 \\
0 \\
0\end{array}$ & 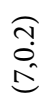 & $\begin{array}{l}\text { f! } \\
\stackrel{1}{1} \\
\dot{f}\end{array}$ & 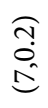 & $\begin{array}{l}\text { F! } \\
\stackrel{0}{0} \\
\hat{\omega}\end{array}$ & 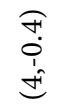 & 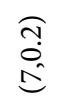 & 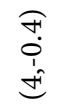 & 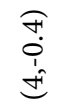 & $\begin{array}{l}\text { f! } \\
\text { ì } \\
\text { \&ी }\end{array}$ & 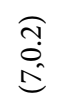 & $\begin{array}{l}\text { f! } \\
\text { 1. } \\
\text { d }\end{array}$ \\
\hline ชี & $\begin{array}{l}\widehat{\widehat{T}} \\
\hat{1} \\
\hat{\mathfrak{d}}\end{array}$ & \begin{tabular}{l} 
f̦ \\
$\stackrel{1}{1}$ \\
\multirow{4}{*}{}
\end{tabular} & 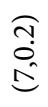 & $\begin{array}{l}\text { f̦ } \\
\stackrel{1}{1} \\
\dot{1}\end{array}$ & $\begin{array}{l}\overparen{F} \\
0 \\
0 \\
0\end{array}$ & 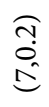 & $\begin{array}{l}\text { f! } \\
\stackrel{1}{1} \\
\dot{J}\end{array}$ & $\begin{array}{l}\widehat{T} \\
\hat{1} \\
\hat{d} \\
\hat{d}\end{array}$ & 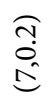 & 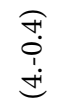 & $\begin{array}{l}\text { F! } \\
\stackrel{0}{0} \\
\hat{\omega}\end{array}$ & 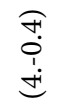 & 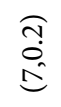 & 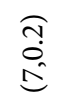 & $\begin{array}{l}\text { f! } \\
\stackrel{1}{1} \\
\dot{J}\end{array}$ & 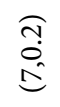 & $\begin{array}{l}\text { กิ } \\
\text { 1. } \\
\text { du }\end{array}$ \\
\hline 2 & 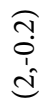 & 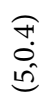 & 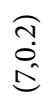 & $\begin{array}{l}\text { f̊. } \\
\text { in } \\
\dot{1}\end{array}$ & 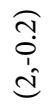 & 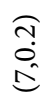 & 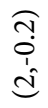 & $\begin{array}{l}\overparen{F} \\
0 \\
0 \\
0\end{array}$ & $\begin{array}{l}\text { f̊. } \\
\stackrel{1}{1} \\
\dot{J}\end{array}$ & 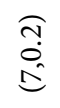 & 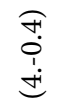 & $\begin{array}{l}\text { กุ } \\
\grave{1} \\
\stackrel{d}{0}\end{array}$ & 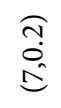 & $\begin{array}{l}\text { f! } \\
\stackrel{1}{1} \\
\text { +̇ }\end{array}$ & 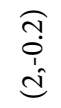 & 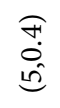 & 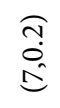 \\
\hline$\vec{a}$ & 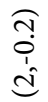 & $\begin{array}{l}\overparen{f} \\
\stackrel{0}{0} \\
\dot{0}\end{array}$ & 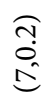 & $\begin{array}{l}\text { fí } \\
\dot{1} \\
\dot{f}\end{array}$ & 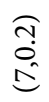 & $\begin{array}{l}\text { f: } \\
\dot{1} \\
\dot{H}\end{array}$ & 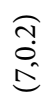 & $\begin{array}{l}\overparen{f} \\
\stackrel{0}{0} \\
\dot{0}\end{array}$ & $\begin{array}{l}\text { f̦ } \\
\dot{1} \\
\dot{H}\end{array}$ & $\begin{array}{l}\text { กิ } \\
\text { 1. } \\
\text { ĉd }\end{array}$ & $\begin{array}{l}\text { f! } \\
\stackrel{1}{1} \\
\stackrel{+}{+}\end{array}$ & $\begin{array}{l}\overparen{f} \\
\stackrel{0}{0} \\
\stackrel{0}{0}\end{array}$ & 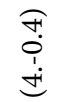 & 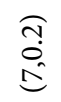 & $\begin{array}{l}\text { f! } \\
\stackrel{1}{1} \\
\stackrel{+}{+}\end{array}$ & $\begin{array}{l}\text { f! } \\
\stackrel{0}{0} \\
\stackrel{0}{0}\end{array}$ & 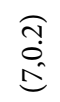 \\
\hline
\end{tabular}

Table 6. The index evaluation value by expert 2

\begin{tabular}{|c|c|c|c|c|c|c|c|c|c|c|c|c|c|c|c|c|c|}
\hline & B1 & B2 & B3 & B4 & B5 & B6 & B7 & B8 & B9 & B10 & B11 & B12 & B13 & B14 & B15 & B16 & B17 \\
\hline च & 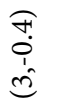 & $\begin{array}{l}\widehat{0} \\
0 \\
\dot{0}\end{array}$ & $\begin{array}{l}\hat{n} \\
\hat{i} \\
1 \\
\hat{\infty}\end{array}$ & $\begin{array}{l}\text { త্ } \\
\dot{1} \\
\dot{t}\end{array}$ & $\begin{array}{l}\widehat{0} \\
0 \\
\dot{0}\end{array}$ & $\begin{array}{l}\text {-’ } \\
\dot{1} \\
\dot{J}\end{array}$ & $\begin{array}{l}\text { f! } \\
\dot{1} \\
1 \\
\hat{C}\end{array}$ & $\begin{array}{l}\widehat{0} \\
\dot{0} \\
\dot{0}\end{array}$ & $\begin{array}{l}\stackrel{f}{*} \\
0 \\
0 \\
0\end{array}$ & $\begin{array}{l}\widehat{n} \\
0 \\
1 \\
0 \\
0\end{array}$ & $\begin{array}{l}\hat{n} \\
\stackrel{0}{=} \\
=\end{array}$ & 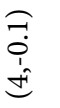 & $\begin{array}{l}\widehat{0} \\
0 \\
0 \\
0\end{array}$ & $\begin{array}{l}\widehat{3} \\
0 \\
1 \\
\hat{0}\end{array}$ & 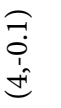 & $\begin{array}{l}\text { f! } \\
\vdots \\
1 \\
0 \\
0\end{array}$ & $\begin{array}{l}\widehat{0} \\
\dot{0} \\
\dot{0}\end{array}$ \\
\hline$\widetilde{\varepsilon}$ & $\begin{array}{l}\text { f! } \\
0 \\
1 \\
0 \\
0\end{array}$ & $\begin{array}{l}\widehat{0} \\
\hat{0} \\
0\end{array}$ & 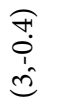 & $\begin{array}{l}\overparen{f} \\
\stackrel{0}{0} \\
\hat{\theta}\end{array}$ & $\begin{array}{l}\widehat{m} \\
0 \\
i \\
\hat{\infty} \\
\hat{\infty}\end{array}$ & $\begin{array}{l}\text {-’ } \\
\dot{1} \\
\dot{J}\end{array}$ & $\begin{array}{l}\text {-’ } \\
\dot{1} \\
\dot{t}\end{array}$ & $\begin{array}{l}\widehat{\tilde{n}} \\
\stackrel{0}{=} \\
=\end{array}$ & $\begin{array}{l}\widehat{3} \\
0 \\
0 \\
0\end{array}$ & $\begin{array}{l}\overparen{F} \\
\stackrel{0}{\theta} \\
\hat{\theta}\end{array}$ & $\begin{array}{l}\text { - } \\
\dot{0} \\
\dot{t}\end{array}$ & $\begin{array}{l}\overparen{f} \\
0 \\
0 \\
0\end{array}$ & $\begin{array}{l}\widehat{n} \\
0 \\
1 \\
\hat{\infty}\end{array}$ & $\begin{array}{l}\overparen{f} \\
0 \\
0 \\
0\end{array}$ & $\begin{array}{l}\text { f! } \\
\text { 1. } \\
\text { ؛́ }\end{array}$ & $\begin{array}{l}\widehat{n} \\
\stackrel{0}{=} \\
=\end{array}$ & $\begin{array}{l}\text {-’ } \\
\dot{0} \\
\dot{t}\end{array}$ \\
\hline 2 & $\begin{array}{l}\hat{\tilde{n}} \\
\stackrel{0}{=}\end{array}$ & $\begin{array}{l}\text { - } \\
\dot{0} \\
\dot{t}\end{array}$ & $\begin{array}{l}\text { - } \\
\dot{0} \\
\dot{t}\end{array}$ & $\begin{array}{l}\text { f! } \\
0 \\
1 \\
\hat{n}\end{array}$ & $\begin{array}{l}\widehat{\tilde{n}} \\
\stackrel{0}{=}\end{array}$ & $\begin{array}{l}\widehat{3} \\
0 \\
0 \\
0\end{array}$ & $\begin{array}{l}\overparen{f} \\
0 \\
0 \\
0\end{array}$ & $\begin{array}{l}\text { - } \\
\dot{0} \\
\dot{t}\end{array}$ & 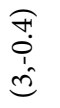 & $\begin{array}{l}\widehat{0} \\
\stackrel{0}{1} \\
\vdots \\
0 \\
0\end{array}$ & $\begin{array}{l}\overparen{f} \\
0 \\
0 \\
0\end{array}$ & $\begin{array}{c}\widehat{a} \\
0 \\
0 \\
0\end{array}$ & $\begin{array}{l}\text { - } \\
\dot{0} \\
\dot{t}\end{array}$ & $\begin{array}{l}\text { ঙ! } \\
0 \\
1 \\
\stackrel{0}{0}\end{array}$ & $\begin{array}{l}\overparen{f} \\
0 \\
\hat{\theta}\end{array}$ & $\begin{array}{l}\widehat{0} \\
0 \\
0 \\
0\end{array}$ & $\begin{array}{l}\overparen{f} \\
0 \\
0 \\
0\end{array}$ \\
\hline J & $\begin{array}{l}\widehat{0} \\
\dot{0} \\
\stackrel{0}{0}\end{array}$ & $\begin{array}{l}\text { - } \\
\dot{0} \\
\dot{t}\end{array}$ & $\begin{array}{l}\widehat{0} \\
\dot{0} \\
\underline{0}\end{array}$ & $\begin{array}{l}\overparen{f} \\
\stackrel{0}{0} \\
\hat{\theta}\end{array}$ & $\begin{array}{l}\widehat{n} \\
\stackrel{0}{\Leftrightarrow} \\
\Leftrightarrow\end{array}$ & 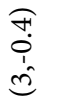 & $\begin{array}{l}\text {-़. } \\
\dot{0} \\
\dot{f}\end{array}$ & $\begin{array}{l}\overparen{f} \\
\stackrel{0}{0} \\
\hat{\theta}\end{array}$ & $\begin{array}{l}\widehat{\overrightarrow{0}} \\
\dot{0} \\
\hat{0}\end{array}$ & $\begin{array}{l}\text { - } \\
\text { 1. } \\
\text { dी }\end{array}$ & $\begin{array}{l}\text { f! } \\
\stackrel{1}{1} \\
\stackrel{0}{0}\end{array}$ & $\begin{array}{l}\hat{n} \\
\hat{1} \\
1 \\
\hat{\infty}\end{array}$ & $\begin{array}{l}\hat{n} \\
0 \\
1 \\
\hat{0}\end{array}$ & $\begin{array}{l}\overparen{f} \\
\stackrel{0}{0} \\
\hat{\theta}\end{array}$ & 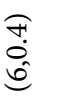 & 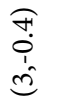 & $\begin{array}{l}\overparen{f} \\
\stackrel{0}{0} \\
\hat{\theta}\end{array}$ \\
\hline
\end{tabular}


Table 7. The index evaluation value by expert 3

\begin{tabular}{|c|c|c|c|c|c|c|c|c|c|c|c|c|c|c|c|c|c|}
\hline & B1 & B2 & B3 & B4 & B5 & B6 & B7 & B8 & B9 & B10 & B11 & B12 & B13 & B14 & B15 & B16 & B17 \\
\hline$\vec{\sigma}$ & $\begin{array}{l}\hat{\theta} \\
\dot{0} \\
\hat{0}\end{array}$ & 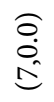 & $\begin{array}{l}\hat{0} \\
\dot{0} \\
\dot{\oplus}\end{array}$ & $\begin{array}{l}\hat{0} \\
\dot{0} \\
\hat{\infty}\end{array}$ & $\begin{array}{l}\text { ô } \\
\dot{0} \\
\stackrel{d}{d}\end{array}$ & $\begin{array}{l}\text { Oे } \\
\dot{0} \\
\dot{+}\end{array}$ & $\begin{array}{l}\hat{0} \\
\dot{0} \\
\hat{d}\end{array}$ & $\begin{array}{l}\hat{0} \\
\dot{0} \\
\hat{0}\end{array}$ & $\begin{array}{l}\hat{0} \\
\dot{0} \\
\hat{0}\end{array}$ & $\begin{array}{l}\hat{o} \\
\dot{0} \\
\mathfrak{d}\end{array}$ & $\begin{array}{l}\hat{0} \\
\dot{0} \\
\Leftrightarrow\end{array}$ & $\begin{array}{l}\hat{\partial} \\
\dot{0} \\
\hat{0}\end{array}$ & $\begin{array}{l}\hat{\partial} \\
\dot{0} \\
\hat{\theta}\end{array}$ & $\begin{array}{l}\hat{0} \\
\dot{0} \\
\stackrel{0}{0}\end{array}$ & $\begin{array}{l}\text { ọ } \\
\dot{0} \\
\stackrel{d}{d}\end{array}$ & 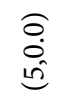 & $\begin{array}{l}\hat{\partial} \\
\dot{0} \\
\stackrel{0}{d}\end{array}$ \\
\hline$\tilde{\Xi}$ & $\stackrel{0}{0}$ & $\begin{array}{l}\text { ọ } \\
\dot{d} \\
\stackrel{i}{c}\end{array}$ & $\begin{array}{l}\hat{0} \\
\dot{0} \\
\dot{\oplus}\end{array}$ & $\begin{array}{l}\hat{0} \\
\dot{0} \\
\hat{\theta}\end{array}$ & 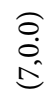 & $\begin{array}{l}\widehat{O} \\
\dot{0} \\
\hat{\infty}\end{array}$ & $\begin{array}{l}\hat{0} \\
\dot{0} \\
\dot{+}\end{array}$ & $\begin{array}{l}\hat{0} \\
\dot{0} \\
\hat{\theta}\end{array}$ & $\begin{array}{l}\hat{0} \\
\dot{0} \\
\hat{\sigma}\end{array}$ & 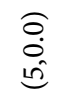 & $\begin{array}{l}\hat{0} \\
\dot{0} \\
\dot{0}\end{array}$ & $\begin{array}{l}\hat{0} \\
\dot{0} \\
\dot{\theta}\end{array}$ & 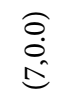 & $\begin{array}{l}\hat{0} \\
\dot{0} \\
\stackrel{0}{0}\end{array}$ & $\begin{array}{l}\hat{0} \\
\dot{0} \\
\stackrel{0}{\ominus}\end{array}$ & 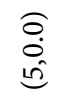 & 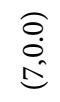 \\
\hline 2 & $\begin{array}{l}\hat{0} \\
\dot{0} \\
\stackrel{0}{=}\end{array}$ & 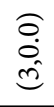 & 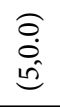 & $\begin{array}{l}\hat{0} \\
0 \\
0 \\
\infty\end{array}$ & 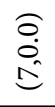 & 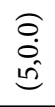 & 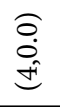 & 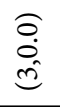 & 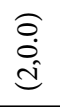 & $\begin{array}{l}\hat{0} \\
\dot{0} \\
\hat{\theta}\end{array}$ & $\begin{array}{l}\hat{0} \\
\dot{0} \\
\stackrel{0}{C}\end{array}$ & $\begin{array}{l}\hat{0} \\
\dot{0} \\
\hat{\infty}\end{array}$ & $\begin{array}{l}\hat{\partial} \\
0 \\
\hat{d}\end{array}$ & 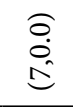 & $\begin{array}{l}\hat{0} \\
\stackrel{0}{0} \\
=\end{array}$ & 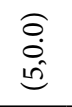 & $\begin{array}{l}\hat{0} \\
0 \\
\infty \\
\infty\end{array}$ \\
\hline 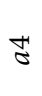 & $\begin{array}{l}\hat{0} \\
\dot{0} \\
\hat{0}\end{array}$ & 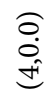 & $\begin{array}{l}\hat{0} \\
\dot{0} \\
\hat{\theta}\end{array}$ & $\begin{array}{l}\hat{0} \\
\dot{0} \\
\hat{0}\end{array}$ & $\begin{array}{l}\stackrel{\partial}{0} \\
\stackrel{0}{\ominus}\end{array}$ & $\begin{array}{l}\hat{0} \\
\dot{0} \\
\hat{\theta}\end{array}$ & 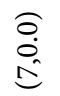 & $\begin{array}{l}\hat{0} \\
\dot{0} \\
\infty \\
\infty\end{array}$ & $\begin{array}{l}\hat{0} \\
\dot{0} \\
\hat{\sigma}\end{array}$ & $\begin{array}{l}\hat{O} \\
\dot{\circ} \\
\dot{d}\end{array}$ & $\begin{array}{l}\hat{0} \\
\dot{\circ} \\
\hat{n}\end{array}$ & $\begin{array}{l}\text { ọ } \\
\dot{0} \\
\stackrel{i}{d}\end{array}$ & $\begin{array}{l}\text { ọ } \\
\dot{0} \\
\dot{\omega}\end{array}$ & $\begin{array}{l}\hat{0} \\
\dot{0} \\
\dot{\theta}\end{array}$ & 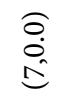 & $\begin{array}{l}\widehat{\partial} \\
\dot{0} \\
\infty \\
\infty\end{array}$ & $\begin{array}{l}\hat{0} \\
\ddot{0} \\
\hat{0}\end{array}$ \\
\hline
\end{tabular}

(2) Integrating each expert's evaluation information, we can get the comprehensive evaluation information shown as table 8.

Table 8. The integrated evaluation matrix

\begin{tabular}{|c|c|c|c|c|c|c|c|c|c|c|c|c|c|c|c|c|c|}
\hline & B1 & B2 & B3 & B4 & B5 & B6 & B7 & B8 & B9 & B10 & B11 & B12 & B13 & B14 & B15 & B16 & B17 \\
\hline$\vec{\sigma}$ & $\begin{array}{l}\overparen{0} \\
\stackrel{0}{+}\end{array}$ & 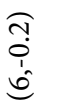 & $\begin{array}{l}\hat{n} \\
\hat{0} \\
\hat{\theta}\end{array}$ & $\begin{array}{l}\text { f! } \\
\dot{1} \\
\dot{1} \\
\dot{0}\end{array}$ & $\begin{array}{l}\text { f̣ } \\
\stackrel{1}{1} \\
\stackrel{f}{+}\end{array}$ & $\begin{array}{l}\stackrel{f}{\circ} \\
\stackrel{0}{+}\end{array}$ & $\begin{array}{l}\overrightarrow{\widehat{0}} \\
\dot{1} \\
\dot{t}\end{array}$ & $\begin{array}{l}\text { F! } \\
\text { i. } \\
\text { فी }\end{array}$ & $\begin{array}{l}\widehat{1} \\
0 \\
0 \\
0 \\
0\end{array}$ & $\begin{array}{l}\text { Ọ. } \\
0 \\
\dot{0}\end{array}$ & $\begin{array}{l}\text { ọ } \\
\dot{0} \\
\hat{d}\end{array}$ & $\begin{array}{l}\text { f! } \\
0 \\
\stackrel{0}{0}\end{array}$ & $\begin{array}{l}\widehat{\overrightarrow{0}} \\
\dot{1} \\
\hat{n}\end{array}$ & $\begin{array}{l}\widehat{0} \\
\overrightarrow{0} \\
\hat{\theta}\end{array}$ & 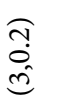 & 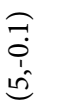 & 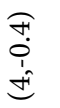 \\
\hline 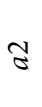 & $\begin{array}{l}\widehat{\Im} \\
\hat{i} \\
1 \\
\hat{d}\end{array}$ & $\begin{array}{l}\text { f̈. } \\
\stackrel{1}{1} \\
\dot{f}\end{array}$ & $\begin{array}{l}\text { f̣ } \\
\dot{1} \\
\dot{1} \\
\hat{n}\end{array}$ & $\begin{array}{l}\widehat{n} \\
0 \\
0 \\
\hat{n}\end{array}$ & 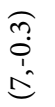 & $\begin{array}{l}\stackrel{f}{\circ} \\
\stackrel{0}{0} \\
\hat{\theta}\end{array}$ & 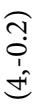 & $\begin{array}{l}\hat{0} \\
\dot{0} \\
\hat{\theta}\end{array}$ & $\begin{array}{l}\overparen{3} \\
\dot{0} \\
\hat{\omega}\end{array}$ & $\begin{array}{l}\text { ọ } \\
\ddot{0} \\
\text { فी }\end{array}$ & $\begin{array}{l}\text { กิ } \\
\vdots \\
1 \\
\hat{n}\end{array}$ & 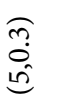 & $\begin{array}{l}\widehat{n} \\
\vdots \\
\text { હ }\end{array}$ & 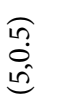 & $\begin{array}{l}\stackrel{f}{:} \\
\stackrel{0}{d}\end{array}$ & 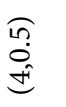 & 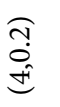 \\
\hline 2 & $\begin{array}{l}\stackrel{\Im}{0} \\
\stackrel{0}{=}\end{array}$ & $\begin{array}{l}\widehat{\Xi} \\
\stackrel{0}{ \pm}\end{array}$ & $\begin{array}{l}\overparen{f} \\
0 \\
0 \\
0\end{array}$ & $\begin{array}{l}\hat{n} \\
0 \\
i \\
\hat{n}\end{array}$ & $\begin{array}{l}\stackrel{\Im}{0} \\
\stackrel{0}{0}\end{array}$ & $\begin{array}{l}\widehat{̃} \\
0 \\
1 \\
\hat{e}\end{array}$ & $\begin{array}{l}\widehat{0} \\
\stackrel{0}{ \pm}\end{array}$ & $\begin{array}{l}\overparen{0} \\
\stackrel{0}{ \pm}\end{array}$ & $\begin{array}{l}\widehat{n} \\
0 \\
i \\
\hat{n} \\
\hat{n}\end{array}$ & 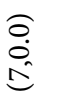 & $\begin{array}{l}\hat{n} \\
0 \\
1 \\
\hat{e}\end{array}$ & 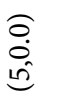 & $\begin{array}{l}\hat{n} \\
0 \\
\vdots \\
\hat{n}\end{array}$ & 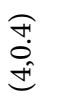 & $\begin{array}{l}\widehat{-} \\
\hat{0} \\
\hat{0}\end{array}$ & $\begin{array}{l}\text { त़ } \\
0 \\
\hat{n}\end{array}$ & 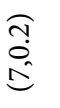 \\
\hline 芯 & $\begin{array}{l}\hat{0} \\
\dot{0} \\
\dot{t}\end{array}$ & $\begin{array}{l}\stackrel{\leftrightarrow}{0} \\
\stackrel{0}{ \pm}\end{array}$ & $\begin{array}{l}\widehat{O} \\
\dot{0} \\
\hat{e}\end{array}$ & \begin{tabular}{l}
$\widetilde{n}$ \\
$\vdots$ \\
\multirow{0}{*}{}
\end{tabular} & $\begin{array}{l}\widehat{a} \\
\tilde{0} \\
\hat{n}\end{array}$ & $\begin{array}{l}\overparen{Z} \\
\stackrel{0}{0} \\
\dot{f}\end{array}$ & $\begin{array}{l}\hat{0} \\
\dot{0} \\
\hat{\theta}\end{array}$ & 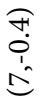 & $\begin{array}{l}\widehat{C} \\
0 \\
\underline{1} \\
\hat{0}\end{array}$ & $\begin{array}{l}\widehat{\hat{n}} \\
\stackrel{0}{0} \\
\hat{n}\end{array}$ & $\begin{array}{l}\overparen{7} \\
0 \\
\hat{0}\end{array}$ & $\begin{array}{l}\text { ọ } \\
\dot{0} \\
\dot{\omega}\end{array}$ & $\begin{array}{l}\text { } \\
\stackrel{0}{0} \\
\stackrel{0}{0}\end{array}$ & $\begin{array}{l}\text { ñ? } \\
0 \\
0 \\
\hat{e}\end{array}$ & $\begin{array}{l}\text { n? } \\
0 \\
1 \\
\hat{\theta}\end{array}$ & $\begin{array}{l}\widehat{n} \\
\stackrel{0}{0} \\
\hat{n}\end{array}$ & $\begin{array}{l}\widehat{\hat{n}} \\
\stackrel{0}{0} \\
\hat{\theta}\end{array}$ \\
\hline
\end{tabular}

(3) Using the entropy weight method to calculate attribute weights

(a) Calculating $u_{i j}$ by formula (7) shown as table 9 .

Table 9. The value of $u_{i j}$

\begin{tabular}{|c|c|c|c|c|c|c|c|c|c|c|c|c|c|c|c|c|c|}
\hline & B1 & B2 & B3 & B4 & B5 & B6 & B7 & B8 & B9 & B10 & B11 & B12 & B13 & B14 & B15 & B16 & B17 \\
\hline J & 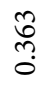 & 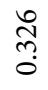 & 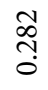 & 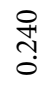 & $\stackrel{n}{\tilde{n}}$ & $\frac{\mathbb{H}}{\stackrel{+}{0}}$ & 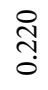 & $\begin{array}{l}\stackrel{\infty}{\infty} \\
\stackrel{1}{0}\end{array}$ & 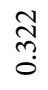 & 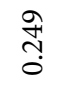 & $\underset{\circlearrowleft}{\stackrel{\widehat{O}}{0}}$ & 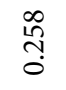 & 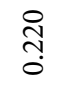 & 犬̊. & 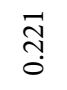 & $\begin{array}{l}\text { f } \\
\text { J }\end{array}$ & $\begin{array}{l}\mathfrak{g} \\
\stackrel{1}{0}\end{array}$ \\
\hline ฮี & 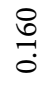 & 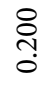 & 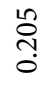 & 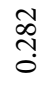 & $\begin{array}{l}\text { ईे } \\
\hat{0}\end{array}$ & $\begin{array}{l}\infty \\
\stackrel{0}{0} \\
0\end{array}$ & $\frac{\vec{J}}{\tilde{\sigma}}$ & 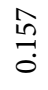 & 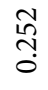 & $\begin{array}{l}\stackrel{\infty}{\sim} \\
\stackrel{0}{0}\end{array}$ & $\begin{array}{l}\infty \\
\stackrel{\infty}{0} \\
0\end{array}$ & $\begin{array}{l}\stackrel{\infty}{\stackrel{n}{n}} \\
\stackrel{0}{0}\end{array}$ & तิ & $\stackrel{\stackrel{\mathscr{H}}{\mathrm{H}}}{\stackrel{0}{\circ}}$ & $\begin{array}{l}\hat{0} \\
\stackrel{1}{0}\end{array}$ & 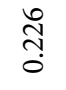 & $\frac{2}{\stackrel{2}{0}}$ \\
\hline 2 & 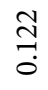 & 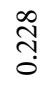 & 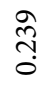 & 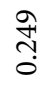 & $\begin{array}{l}\stackrel{丶}{0} \\
0\end{array}$ & $\begin{array}{l}8 \\
\stackrel{\infty}{1} \\
0\end{array}$ & 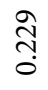 & 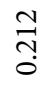 & $\stackrel{\vec{H}}{\stackrel{+}{0}}$ & 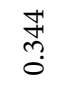 & $\begin{array}{l}\hat{\sigma} \\
\stackrel{n}{0} \\
0\end{array}$ & $\underset{\stackrel{ }{+}}{\stackrel{1}{0}}$ & 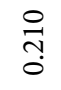 & $\begin{array}{l}\stackrel{H}{\circ} \\
\stackrel{0}{0}\end{array}$ & 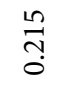 & $\begin{array}{l}\stackrel{8}{\circ} \\
\text { గ̦ }\end{array}$ & 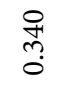 \\
\hline 芯 & $\begin{array}{l}\stackrel{0}{n} \\
\\
0\end{array}$ & 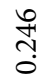 & 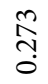 & $\begin{array}{l}\text { సે } \\
\text { ô }\end{array}$ & $\begin{array}{l}\infty \\
\stackrel{\infty}{0} \\
0\end{array}$ & $\frac{\hat{\sigma}}{0}$ & 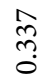 & 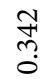 & స్ন & $\begin{array}{l}\stackrel{0}{10} \\
\stackrel{0}{0}\end{array}$ & $\frac{\infty}{\stackrel{2}{0}}$ & 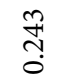 & 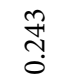 & $\begin{array}{l}\text { ते } \\
\text { ஸे }\end{array}$ & مे & 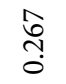 & సิ \\
\hline
\end{tabular}


(b) Calculating the weights of attributes

$$
\begin{aligned}
& W=(0.188,0.032,0.014,0.006,0.100,0.031,0.036,0.077,0.084,0.065 \text {, } \\
& 0.136,0.001,0.030,0.012,0.105,0.004,0.072 \text { ) }
\end{aligned}
$$

(4) Sorting the alternatives using VIKOR method

(a) Calculating the value of the ideal solution $f_{j}^{*}$ and the negative ideal solution $f_{j}^{-}$ of every index (shown as table 10).

\begin{tabular}{|c|c|c|c|c|c|c|c|c|c|c|c|c|c|c|c|c|c|}
\hline & B1 & B2 & B3 & B4 & B5 & B6 & B7 & B8 & B9 & B10 & B11 & B12 & B13 & B14 & B15 & B16 & B17 \\
\hline$\sqrt{4}$ & $\begin{array}{l}\hat{\text { th }} \\
\text { + }\end{array}$ & $\begin{array}{l}\text { f } \\
\infty \\
\text { in }\end{array}$ & 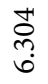 & $\begin{array}{l}\text { Hे } \\
\text { in }\end{array}$ & $\begin{array}{l}\stackrel{H}{R} \\
\text {. }\end{array}$ & $\begin{array}{c}\text { กิ } \\
\text { ભn }\end{array}$ & $\stackrel{\infty}{\stackrel{\infty}{\sigma}}$ & $\begin{array}{l}\text { oे } \\
\text { b }\end{array}$ & 离 & 弇 & $\begin{array}{l}\stackrel{0}{0} \\
6 \\
\text { in }\end{array}$ & $\begin{array}{l}\hat{N} \\
\text { in } \\
\text { in }\end{array}$ & 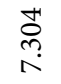 & 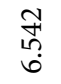 & $\begin{array}{l}0 \\
6 \\
6 \\
i\end{array}$ & $\begin{array}{l}\tilde{N} \\
\text { in }\end{array}$ & 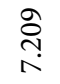 \\
\hline 4 & ָָ & $\begin{array}{l}\vec{\infty} \\
1 \\
\end{array}$ & $\begin{array}{l}8 \\
\text { ถn } \\
+7\end{array}$ & 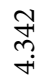 & $\frac{\widetilde{C}}{\dot{m}}$ & $\begin{array}{l}\hat{\text { Un }} \\
\text { + }\end{array}$ & $\begin{array}{l}\stackrel{a}{\infty} \\
\dot{m}\end{array}$ & 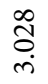 & 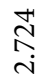 & 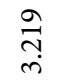 & 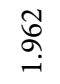 & $\begin{array}{l}\stackrel{2}{\infty} \\
\stackrel{+}{+}\end{array}$ & 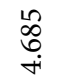 & $\begin{array}{l}\text { ले } \\
+\end{array}$ & 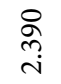 & $\begin{array}{l}\stackrel{2}{\stackrel{f}{+}} \\
+\end{array}$ & 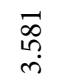 \\
\hline
\end{tabular}

Table 10. The value of $f_{j}^{\star}$ and $f_{j}^{-}$

(b) Calculating the value of $S_{i}$ and $R_{i}$ using formula (12) and (13), $i=1,2, \ldots, m, S_{i}$ is comprehensive evaluation optimal solution, $R_{i}$ is the most bad solution comprehensive evaluation solution.

$$
S=(0.53,0.57,0.62,0.37) \quad R=(0.14,0.16,0.19,0.10)
$$

(c) Calculating value of benefits' ratio made by scheme $Q_{i}$ using formula (14), $i=1,2, \ldots, m$.

$$
Q=(-0.529,-0.743,-1,0)
$$

(d) According to the calculation value $Q$, sort and make a final decision. The scheme of minimum value of the sorting $Q$ is the considered most optimal scheme.

$$
Q_{3} \prec Q_{2} \prec Q_{1} \prec Q_{4}
$$

So choose the a3 candidate as the best solution.

To prove the validity of the method, we use the different method to recalculate the example. Firstly, according to the maximum deviation method, we can calculate the weight,

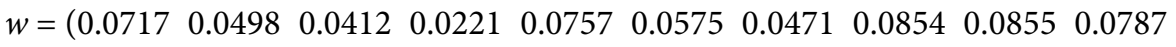

$$
\begin{aligned}
& \left.\begin{array}{lllllll}
0.0896 & 0.0099 & 0.0585 & 0.0490 & 0.0693 & 0.0191 & 0.0898
\end{array}\right) \text {. }
\end{aligned}
$$

Secondly, using the TOPSIS method, we recalculate the example (Hwang, Yoon 1981; Liu 2009). Step 1: Construct the weighted normalized matrix (shown as table 11).

$$
V=\left(v_{i j}\right)_{m \times n}=\left[\begin{array}{cccc}
w_{1} r_{11} & w_{2} r_{12} & \cdots & w_{n} r_{1 n} \\
w_{1} r_{21} & w_{2} r_{22} & \cdots & w_{n} r_{2 n} \\
\cdots & \cdots & \cdots & \cdots \\
w_{1} r_{m 1} & w_{2} r_{m 2} & \cdots & w_{n} r_{m n}
\end{array}\right] .
$$


Table 11. The weighted normalized matrix $\left(v_{i j}\right)_{m \times n}$

\begin{tabular}{|c|c|c|c|c|c|c|c|c|c|c|c|c|c|c|c|c|c|}
\hline & B1 & B2 & B3 & B4 & B5 & B6 & B7 & B8 & B9 & B10 & B11 & B12 & B13 & B14 & B15 & B16 & B17 \\
\hline ह & $\begin{array}{l}\text { ڤे } \\
\text { ஸे } \\
\text { o }\end{array}$ & 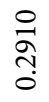 & 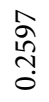 & $\begin{array}{l}\hat{8} \\
8 \\
\vdots \\
0\end{array}$ & $\begin{array}{l}\stackrel{0}{1} \\
\stackrel{\text { }}{0}\end{array}$ & $\begin{array}{l}\stackrel{3}{n} \\
\stackrel{\text { ñ }}{o}\end{array}$ & 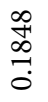 & 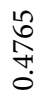 & $\begin{array}{l}n \hat{2} \\
\hat{1} \\
\hat{n} \\
0\end{array}$ & 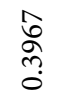 & $\begin{array}{l}\hat{n} \\
\stackrel{0}{0}\end{array}$ & 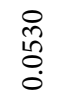 & 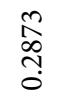 & $\begin{array}{l}\text { के } \\
\text { సે } \\
\text { ஸे }\end{array}$ & 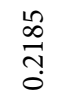 & 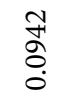 & 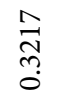 \\
\hline $\mathcal{\varepsilon}$ & $\begin{array}{l}\stackrel{+}{\infty} \\
\stackrel{1}{*} \\
\stackrel{0}{0}\end{array}$ & $\begin{array}{l}\stackrel{\infty}{\infty} \\
\stackrel{0}{0}\end{array}$ & $\begin{array}{l}\vec{\sigma} \\
\stackrel{0}{0}\end{array}$ & $\begin{array}{c}\infty \\
\stackrel{\infty}{二} \\
\end{array}$ & $\begin{array}{l}+1 \\
\text { م⿱ } \\
\text { in } \\
0\end{array}$ & $\begin{array}{l}\hat{2} \\
\hat{0} \\
0 \\
0\end{array}$ & $\begin{array}{l}\stackrel{2}{\curvearrowright} \\
\stackrel{-}{0}\end{array}$ & 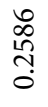 & 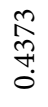 & 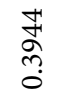 & 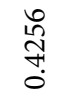 & $\begin{array}{l}\text { ते } \\
\text { है } \\
\text { ○े }\end{array}$ & \begin{tabular}{l}
\multirow{I}{I}{} \\
$\stackrel{+}{0}$
\end{tabular} & 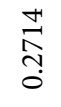 & $\begin{array}{l}\hat{n} \\
0 \\
0 \\
0\end{array}$ & $\begin{array}{l}8 \\
\stackrel{0}{0} \\
\stackrel{0}{0} \\
\stackrel{0}{0}\end{array}$ & 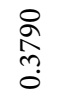 \\
\hline 2 & $\begin{array}{l}0 \\
\stackrel{2}{a} \\
\dot{0} \\
\dot{0}\end{array}$ & $\begin{array}{l}\text { ஸे } \\
\stackrel{\text { }}{0}\end{array}$ & $\begin{array}{l}\stackrel{n}{\hat{~}} \\
\text { ָ̦ }\end{array}$ & 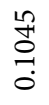 & 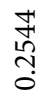 & 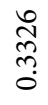 & $\begin{array}{l}\stackrel{\overbrace{}}{\leftrightarrows} \\
\stackrel{0}{\circ}\end{array}$ & $\begin{array}{l}\infty \\
\stackrel{\infty}{\infty} \\
\stackrel{m}{+} \\
0\end{array}$ & $\begin{array}{l}\text { ते } \\
\text { ָ๋ }\end{array}$ & $\begin{array}{l}\stackrel{2}{\infty} \\
+1 \\
\text { مै } \\
0\end{array}$ & 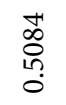 & 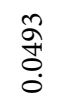 & 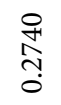 & $\begin{array}{l}\stackrel{\circ}{n} \\
\text { ก̦ } \\
0\end{array}$ & $\frac{\tilde{N}}{\tilde{\sigma}}$ & $\begin{array}{l}\bar{\sigma} \\
\text { oे } \\
0\end{array}$ & $\begin{array}{l}\stackrel{0}{\circ} \\
\text { f̛ } \\
\stackrel{0}{0}\end{array}$ \\
\hline Z & 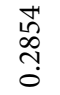 & 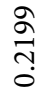 & $\begin{array}{l}\stackrel{a}{n} \\
\stackrel{n}{0}\end{array}$ & $\begin{array}{l}\bar{\varnothing} \\
8 \\
0 \\
0\end{array}$ & 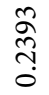 & 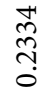 & $\begin{array}{l}\tilde{N} \\
\stackrel{\infty}{0} \\
\stackrel{1}{0}\end{array}$ & $\begin{array}{l}\text { Zु } \\
\stackrel{0}{0} \\
0\end{array}$ & 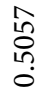 & $\begin{array}{l}\stackrel{n}{\tilde{n}} \\
\stackrel{n}{n} \\
0\end{array}$ & 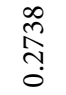 & 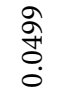 & $\begin{array}{l}\infty \\
\stackrel{\infty}{m} \\
0\end{array}$ & 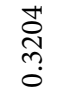 & $\begin{array}{l}\stackrel{H}{\sigma} \\
\text { ஸे }\end{array}$ & $\begin{array}{l}\stackrel{a}{\circ} \\
\stackrel{0}{0}\end{array}$ & $\begin{array}{l}\infty \\
10 \\
n \\
n \\
0 \\
0\end{array}$ \\
\hline
\end{tabular}

Step 2: Determine the ideal solution and negative ideal solution of the object (shown as table 12).

Table 12. The ideal solution and negative ideal solution of the object

\begin{tabular}{|c|c|c|c|c|c|c|c|c|c|c|c|c|c|c|c|c|c|}
\hline & B1 & B2 & B3 & B4 & B5 & B6 & B7 & B8 & B9 & B10 & B11 & B12 & B13 & B14 & B15 & B16 & B17 \\
\hline$\stackrel{+}{s}$ & $\begin{array}{l}\text { ळे } \\
\text { సે }\end{array}$ & 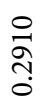 & 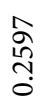 & $\stackrel{\stackrel{\infty}{\infty}}{\exists}$ & $\begin{array}{l}\text { H } \\
\text { 号 } \\
0\end{array}$ & 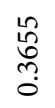 & 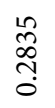 & 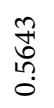 & $\begin{array}{l}\text { مू } \\
\hat{h} \\
\hat{n} \\
0\end{array}$ & $\begin{array}{l}\text { oे } \\
\text { †े } \\
\text { th } \\
0\end{array}$ & $\begin{array}{l}+ \\
\infty \\
0 \\
0 \\
0\end{array}$ & $\begin{array}{l}\stackrel{D}{0} \\
\tilde{0} \\
0 \\
0\end{array}$ & 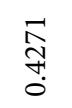 & 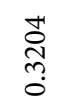 & $\begin{array}{l}\text { ळે } \\
\text { ळે }\end{array}$ & $\begin{array}{l}\stackrel{a}{0} \\
\stackrel{0}{0}\end{array}$ & $\begin{array}{l}\stackrel{0}{~} \\
\text { fo } \\
\stackrel{0}{0}\end{array}$ \\
\hline $1-2$ & $\begin{array}{l}0 \\
\stackrel{0}{\circ} \\
0 \\
0\end{array}$ & $\begin{array}{l}\frac{\infty}{2} \\
\stackrel{1}{0}\end{array}$ & $\begin{array}{l}\vec{\sigma} \\
\overrightarrow{0} \\
\overrightarrow{0}\end{array}$ & $\begin{array}{l}\overrightarrow{0} \\
\text { ᄋ̆ } \\
0\end{array}$ & $\begin{array}{c}\text { ָे } \\
\text { ஸ̦ }\end{array}$ & $\begin{array}{l}\stackrel{+}{N} \\
\stackrel{n}{0}\end{array}$ & $\begin{array}{l}\stackrel{2}{ล} \\
\stackrel{0}{0}\end{array}$ & 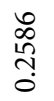 & 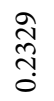 & 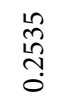 & $\begin{array}{l}\text { in } \\
\stackrel{1}{0}\end{array}$ & 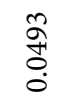 & $\begin{array}{l}\stackrel{+}{\stackrel{1}{1}} \\
\text { N̦ }\end{array}$ & \begin{tabular}{l}
$\stackrel{0}{n}$ \\
\multirow{n}{0}{}
\end{tabular} & $\begin{array}{l}\hat{n} \\
0 \\
\overrightarrow{0}\end{array}$ & $\begin{array}{l}\stackrel{0}{0} \\
\infty \\
0 \\
0\end{array}$ & 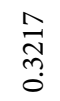 \\
\hline
\end{tabular}

Step 3: Calculate the distance, determine the relative proximity and sort the program.

$$
\begin{gathered}
d_{i}^{+}=\left[\sum_{j=1}^{n}\left(v_{i j}-v_{j}^{+}\right)^{2}\right]^{1 / 2} i=1,2, \cdots, m, \\
d_{i}^{-}=\left[\sum_{j=1}^{n}\left(v_{i j}-v_{j}^{-}\right)^{2}\right]^{1 / 2} \\
C_{i}=\frac{d_{i}^{+}}{d_{i}^{+}+d_{i}^{-}} \cdot(i=1,2, \cdots, m), \\
C_{i}=(0.5670,0.5102,0.4263,0.6096) .
\end{gathered}
$$

According to the size of the relative proximity, we can evaluate the sort merits.

The smaller $C_{i}$ is, the better the program is.

$$
C_{3}<C_{2}<C_{1}<C_{4}
$$

So, the best selection is $a 3$. 
It is the same result for VIKOR and TOPSIS methods, but these two methods have their own characteristics (Opricovic, Tzeng 2004). TOPSIS method is based on the principle that the optimal point should have the shortest distance from the positive ideal solution (PIS) and the farthest from the negative ideal solution (NIS). Therefore, this method is suitable for cautious (risk avoider) decision maker(s), because the decision maker(s) might like to have a decision which not only makes as much profit as possible, but also avoids as much risk as possible. Besides, computing the optimal point in the VIKOR is based on the particular measure of "closeness" to the PIS. Therefore, it is suitable for those situations in which the decision maker wants to have maximum profit and the risk of the decisions is less important for him.

\section{Conclusions}

In the context of economic globalization, human resources had become the key of company's competitive. Human resources managers are the organizers and implementers of human resources management and play a decisive role in human resource management. The levels of human resources managers' competency play a decisive role in whether is effective among human resources management. For the characteristics of human resources managers' competency, this paper raised evaluation of human resources managers' competency based on multi-granularity linguistic variables and VIKOR method, firstly built human resources manager evaluation system based on competency, and by using the concept of two-semantic put multi-granularity evaluation information of different experts into the same granularity of evaluation information. Then we aggregated the evaluation information of each expert to the comprehensive evaluation value, weighted every evaluation indicator by entropy method, get the evaluation value on the ideal solution and negative ideal solution by VIKOR. At last we determined the sort merits of every evaluation objects according to the size of interest rate. Finally by an enterprise application example, we illustrated the evaluation procedures and validity of the model, showed that the method was easy to operate, easy to promote the use. Besides, to different staff of the company, such as position characteristic of production staff, technical staff, management staff, it can separately make different evaluation index system and make evaluation order to these person. Compared with the past quantitative evaluation, this method used the qualitative language evaluation directly and it's easy to use. But during the actual use, evaluation index may be both qualitative and quantitative indicators. The evaluation of this situation will be further studied.

\section{Acknowledgement}

This paper is supported by the National Natural Science Foundation of China (No. 71271124), the Humanities and Social Sciences Research Project of Ministry of Education of China (No. 10YJA630073 and No.09YJA630088), the Natural Science Foundation of Shandong Province (No.ZR2011FM036), and Graduate education innovation projects of Shandong Province (SDYY12065). The author also would like to express appreciation to the anonymous reviewers and Managing Editor Jonas Šaparauskas for their very helpful comments that improved the paper. 


\section{References}

Castrogiovanni, G. J.; Kidwell, R. E. 2010. Human resource management practices affecting unit managers in franchise networks, Human Resource Management 49(2): 225-239.

http://dx.doi.org/10.1002/hrm.20345

Chen, W. S. 2006. Research on human resources manager competency model of China Company, Economic Management (2): 55-63.

Chu, M. T; Shyu, J.; Tzeng, G. H.; Khosla, R. 2007. Comparison among three analytical methods for knowledge communities group-decision analysis, Expert Systems with Applications 33(4): 1011-1024. http://dx.doi.org/10.1016/j.eswa.2006.08.026

Davidson, M. C. G.; Wang, Y. 2011. Sustainable labor practices? Hotel human resource managers views on turnover and skill shortages, Journal of Human Resources in Hospitality \& Tourism 10(3): 235-253. http://dx.doi.org/10.1080/15332845.2011.555731

Gu, Q. X.; Zhu, M. 2001. Competence study of human resources professionals, Human Resource Development of China 9: 24-26.

Han, Z. S.; Liu, P. D. 2011. A fuzzy multi-attribute decision-making method under risk with unknown attribute weights, Technological and Economic Development of Economy 17(2): 246-258. http://dx.doi.org/10.3846/20294913.2011.580575

Hashim, J. 2010. Human resource management practices on organizational commitment: the Islamic perspective, Personnel Review 39(6): 785-799. http://dx.doi.org/10.1108/00483481011075611

Herrera, F.; Martinez, L. 2000. A 2 - tuple fuzzy linguistic representation model for computing with words, IEEE Transactions on Fuzzy Systems 8(6): 746-752. http://dx.doi.org/10.1109/91.890332

Herrera, F.; Martinez, L. 2001. A model based on linguistic 2 - tuples for dealing with multi-granularity hierarchical linguistic contexts in multi-expert decision-making, IEEE Transactions on Systems, Man and Cybernetics-Part B: Cybernetics 31(2): 227-234. http://dx.doi.org/10.1109/3477.915345

Herrera, F.; Martinez, L.; Sanchez, P. J. 2005. Managing non-Homogeneous information in group decision making, European Journal of Operational Research 166(11): 115-132.

http://dx.doi.org/10.1016/j.ejor.2003.11.031

Hwang, C. L.; Yoon, K. L. 1981. Multiple attribute decision making-methods and applications. New York: Springer-Verlag. http://dx.doi.org/10.1007/978-3-642-48318-9

Liu, C. G.; Zhao, S. M.; Huang, F. H. 2009. The study of human resources manager competency, Modern Management Science (1): 6-8.

Liu, P. D. 2009. Multi-attribute decision-making method research based on interval vague set and TOPSIS method, Technological and Economic Development of Economy 15(3): 453-463.

http://dx.doi.org/10.3846/1392-8619.2009.15.453-463

Liu, P. D.; Zhang, X. 2011. Investigation into evaluation of agriculture informatization level based on Two-Tuple, Technological and Economic Development of Economy 17(1): 74-86. http://dx.doi.org/10.3846/13928619.2011.554007

Ma, X. Q.; Cai, H. Q. 2007.Top ten human resource manager competency, Science-Technology and Management (1): 107-110.

Mussari, R.; Ruggiero, P. 2010. Public managers' performance evaluation systems and public value creation: behavioral and economic aspects, International Journal of Public Administration 33(11): 541-548. http://dx.doi.org/10.1080/01900692.2010.507115

Opricovic, S. 1998. Multicriteria optimization of civil engineering systems. Belgrade: Faculty of Civil Engineering (in Serbian).

Opricovic, S.; Tzeng, G. H. 2002. Multicriteria planning of post-earthquake sustainable reconstruction, Computer-Aided Civil and Infrastructure Engineering, 17(3): 211-220.

http://dx.doi.org/10.1111/1467-8667.00269 
Opricovic, S.; Tzeng, G. H. 2004.Compromise solution by MCDM methods: a comparative analysis of VIKOR and TOPSIS, European Journal of Operational Research 156(2): 445-455. http://dx.doi.org/10.1016/S0377-2217(03)00020-1

Opricovic, S.; Tzeng, G. H. 2007. Extended VIKOR method in comparison with outranking methods, European Journal of Operational Research 178(2):514-529. http://dx.doi.org/10.1016/j.ejor.2006.01.020

Sharabi, M. 2010. HR manager leadership in quality improvement in a college environment, Quality Assurance in Education 18(4): 317-327. http://dx.doi.org/10.1108/09684881011079161

Spencer, L. M.; McClelland, D. C.; Spencer, S. 1994. Competency assessment methods: history and state of the art. Hay-McBer Research Press.

Ulrich, D.; Brockbank, W.; Yeung, A. K.; Lake, D. G. 1995. Human resource competencies: an empirical assessment, Human Resource Management 134: 473-495. http://dx.doi.org/10.1002/hrm.3930340402

Wang, M. K.; Hwang, K. P. 2011. Key factors for the successful evaluation and screening of managers of the intellectual property rights speciality, Expert Systems with Applications 38(9): 10794-10802. http://dx.doi.org/10.1016/j.eswa.2011.02.002

Wang, X. H. 2007. Competency-based evaluation system of human resources. Master Thesis: Lanzhou University of Technology. 65-110.

Yu, P. L. 1973. A class of solutions for group decision problems, Management Science 19(8): 936-946. http://dx.doi.org/10.1287/mnsc.19.8.936

Zavadskas, E. K.; Turskis, Z. 2011. Multiple criteria decision making (MCDM) methods in economics: an overview, Technological and Economic Development of Economy 17(2): 397-427. http://dx.doi.org/10.3846/20294913.2011.593291

Zeleny, M. 1982. Multiple Criteria Decision Making. New York: McGraw-Hill.

Zhang, L. X.; Wang, J.; Liu, J. 2005. The subjective and objective evaluation of human resources manager competency, Journal of Northeastern University (Natural Science) 26(11): 1119-1122.

Zhao, F. F. 2008. Competency-based personnel selection fuzzy multiple criteria decision making - an example of human resource manager selection, Science Technology and Management 10(6): 104-106.

Zhou, Y.; Zhang, M. Q. 2009. Fuzzy comprehensive evaluation and empirical study of human resources manager based competency, Science and Technology Management Research (8): 490-493.

Peide LIU. Born in China, 1966. Obtained his bachelor and master degrees in Electronic Technology in the Southeast University, and obtained doctor degree in Information Management in Beijing Jiaotong University. Now he is a full-time professor in Shandong University of Finance and Economics and assistant director of the Enterprise's Electronic-commerce Engineering Research Center of Shandong. His main research interests are technology and information management, decision support and electronic-commerce.

Xingying WU. Born in China, 1990. Obtained her bachelor degree in Electronic-Commerce in the Shandong Economic University. Now she is doing the research work for master degree in Shandong University of Finance and Economics. Her main research interests are information management, decision support and electronic-commerce. 